\title{
An intermodulation products generator for predistortion of base station HPAs
}

\author{
M. X. Xiao, S. W. Cheung and T. I. Yuk \\ The Department of Electrical and Electronic Engineering, the University of Hong Kong \\ mxxiao@eee.hku.hk, swcheung@eee.hku.hk, tiyuk@eee.hku.hk
}

\begin{abstract}
This paper presents the design of an intermodulation (IM) products generator for predistortion of high-power amplifiers (HPAs) used in the base stations of cellular mobile systems. The IM products generator composes of two simple mixers constructed with Schottky diodes, has a simple structure and requires no external de bias voltage. Simulation and measurement results show that the generator has a low conversion loss and low spurious frequency components. Studies of the IM3 generator for predistortion of a practical base station HPA are also carried out by simulation and measurements. Measurement result of a two-tone test shows that the predistorter can suppress the $3^{\text {rd }}$ intermodulation distortion (IMD) products by $15 \mathrm{~dB}$.
\end{abstract}

Index terms - High Power Amplifier, Intermodulation distortion, Intermodulation products generator, Mixer, RF Analog Predistortion

\section{I.INTRODUCTION}

Analog RF predistortion has the advantages of simple and low cost to implement and has a much wider bandwidth than digital predistortion, so it is more suitable for HPAs in the base stations of cellular mobile system. An RF predistorter has a characteristic complementary to that of the RF HPA $[1,2]$ and can be implemented by generating either the harmonicfrequency signals $[1,3,4]$ or the inband intermodulation (IM) products [5-9]. The harmonic-frequency technique has an advantage of low insertion loss [4], but the bandwidth of the HPA will block the high-order harmonic-frequency signals, making the technique impractical to use for high frequency systems. In general, there are two ways, i.e. using the difference-frequency signal or the sum-frequency signal, to generate the inband IM products for HPA predistortion $[5,6]$. In the microwave frequency systems, the difference-frequency signal with a relatively low frequency will be blocked by the HPAs. The sum-frequency signal with twice of the maximum frequency is not an easy-to-handle signal, particularly when generating IM products with higher orders for intermodulation distortion (IMD) products cancellation.

This paper proposes to use an IM products generator composing of two mixers to avoid the mentioned problems for RF HPA predistortion. The mixers are constructed with Schottky diodes: a single-balanced mixer to obtain the down converted difference-frequency signals and a single-ended mixer to generate the wanted IM3 products. Simulation and measurement results on single tone tests show that the mixers have low conversion losses and low spurious frequency components. RF predistortion usually assumes a real polynomial expression to model the HPA. In this paper, a complex polynomial is used to model the HPA and a quadrature predistorter is used for predistortion. Studies are carried out using simulation and measurements. Experimental results using a practical HPA show that, for a two-tone signal, the predistorter can suppress the $3^{\text {rd }}$-intermodulation distortion (IMD) products by $15 \mathrm{~dB}$.

\section{MODEL OF HIGH-POWER AMPLIFIER (HPA)}

The nonlinear process of an HPA can be represented by:

$$
y=A(x) e^{j \phi(x)}=A(x) \cos (\phi(x))+j A(x) \sin (\phi(x))
$$

where $\mathrm{x}$ is the input signal, $\mathrm{y}$ is the output signals, $A($.$) and$ $\phi($.$) are the amplitude-amplitude (AM-AM) and amplitude-$ phase (AM-PM) distortion effects, respectively, of the HPA. Using a $3^{\text {rd }}$-order polynomial representation, (1) can be approximated by:

$$
y=\left(a_{1} x+a_{2} x^{2}+a_{3} x^{3}\right)+j\left(b_{1} x+b_{2} x^{2}+b_{3} x^{3}\right)
$$

where $a_{1}, a_{2}, a_{3}, b_{1}, b_{2}, b_{3}$ are the coefficients of the complex polynomial. Since the even-order terms produce IM products which are outside the HPA operating frequency band and do not contribute to the inband distortion, we can consider only the odd-order terms in (2).

\section{INTERMODULATION PRODCUTS GENERATOR}

Here, we describe how to generate the 3rd-order intermodulation (IM3) signals to suppress the 3rd-order intermodulation distortion (IMD) products introduced by an HPA. The approach can be extended to suppress higher order IMD products. Figure 1 shows our proposed IM3 generator, consisting of 2 mixers, where RF\#1, LO\#1, RF\#2 and LO\#2 are input ports, IF\#1 and IF\#2 are output ports, and $\mathrm{RF}_{1}, \mathrm{LO}_{1}$, $\mathrm{IF}_{1}, \mathrm{RF}_{2}, \mathrm{LO}_{2}$ and $\mathrm{IF}_{2}$ are the signals in these ports respectively.

To generate the IM3 signals, $\cos \left(2 \omega_{2}-\omega_{1}\right) t$ and $\cos \left(2 \omega_{1}-\omega_{2}\right) t$, a two-tone signal, $\cos \left(\omega_{1} t\right)+\cos \left(\omega_{2} t\right)$, is used to feed to the input ports, RF\#1 and LO\#1, of Mixer \#1 to generate the difference-frequency signal, $\cos \left(\omega_{2}-\omega_{1}\right) t$, and 
other unwanted signals at the output port IF\#1. The direct current (DC) component and the unwanted signals are blocked by a capacitor and low-pass filter, respectively. The signal, $\cos \left(\omega_{2}-\omega_{1}\right) t$, from the output port IF\#1 is fed to the input port RF\#2 of Mixer \#2. The input port LO\#2 is fed with the same two-tone signal $\cos \left(\omega_{1} t\right)+\cos \left(\omega_{2} t\right)$ which is mixed with $\cos \left(\omega_{2}-\omega_{1}\right) t$ in Mixer \#2 to generate the wanted IM3 signal, $\cos \left(2 \omega_{1}-\omega_{2}\right) t+\cos \left(2 \omega_{2}-\omega_{1}\right) t$, at the output port IF\#2. In addition to the wanted signal, the unwanted signals are also generated but easily removed as described later.

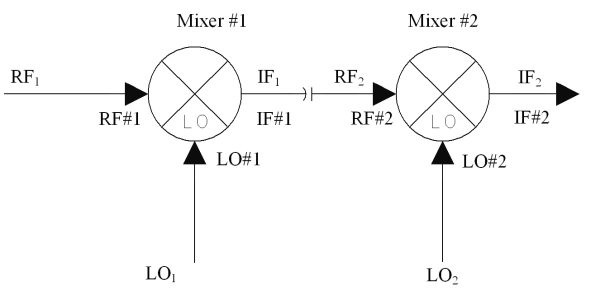

Fig. 1 IM3 generator using mixers

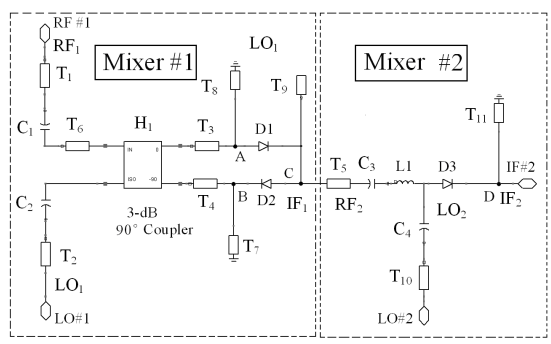

Fig. 2 Circuit of IM3 generator

The actual circuit of the IM3 generator is shown in Fig. 2. Mixer \#1 is a single-balanced mixer. $\mathrm{C}_{1}$ and $\mathrm{C}_{2}$ are the filter capacitors used to block the $\mathrm{DC}$ component, and $\mathrm{T}_{1}$ and $\mathrm{T}_{2}$ are $50-O h m$ transmission lines with equal length serving as the input ports, $\mathrm{RF} \# 1$ and $\mathrm{LO} \# 1$, to which the signals $\mathrm{RF}_{1}$ and $\mathrm{LO}_{1}$, respectively, are fed. $\mathrm{T}_{6}$ is a quarter-wave transmission line at the frequency of $R F_{1}$, so the signal $R_{1}$ at the input of the $3-\mathrm{dB} 90^{\circ}$ hybrid coupler $\left(\mathrm{H}_{1}\right)$ has a $90^{\circ}$ phase delay. At the balanced ports of the coupler, the signals $\mathrm{RF}_{1}$ and $\mathrm{LO}_{1}$ have the same phase in $\mathrm{T}_{4}$, but a phase difference of $180^{\circ}$ in $\mathrm{T}_{3}$. An antipodal diode pair, using Schottky diodes D1 and D2 (as the mixing elements), is connected to the outputs of the coupler. The mixing IF components in each diode element with equal phase are combined together to form the output signal $\mathrm{IF}_{1}$ and those with a phase difference of $180^{\circ}$ are cancelled off. $\mathrm{T}_{7}$ and $\mathrm{T}_{8}$ are quarter-wave short-circuited lines at $\mathrm{RF}_{1}$ frequency. Assuming that the frequencies of $\mathrm{RF}_{1}$ and $\mathrm{LO}_{1}$ are close to each other, so points $\mathrm{A}$ and $\mathrm{B}$ are seen as an open circuit by the signals $\mathrm{RF}_{1}$ and $\mathrm{LO}_{1}$ which are fed to the diodes for mixing. It is noted that the DC signal produced by the mixing process is used to bias the diodes D1 and D2. The signal $\mathrm{IF}_{1}$, being at a much lower frequency, sees points $\mathrm{A}$ and $\mathrm{B}$ as a short circuit. $\mathrm{T}_{9}$ is a quarter-wave open-circuited transmission line also at the frequency of $R F_{1}$, so point $C$ is seen as a short circuit for signals $\mathrm{RF}_{1}$ and $\mathrm{LO}_{1} \cdot \mathrm{T}_{3}$ and $\mathrm{T}_{4}$ are transmission lines used for soldering components. In addition, $T_{3} \& T_{8}$ and $T_{4} \& T_{7}$ also form the matching networks for diodes $\mathrm{D} 1$ and $\mathrm{D} 2$, respectively, to reduce the mixer's conversion loss [10].

Mixer \#2 is a single-ended mixer. The output signal $\mathrm{IF}_{1}$ from Mixer \#1 is fed via $T_{5}$ to the input port $R F \# 2$ and becomes the input signal $\mathrm{RF}_{2}$. The capacitor $\mathrm{C}_{3}$ and inductor $\mathrm{L}_{1}$ form a tuned circuit to block the DC signal and pass the $\mathrm{RF}_{2}$ signal to diode $\mathrm{D} 3$. The tuned circuit also blocks the highfrequency signals $\mathrm{LO}_{2}$ and $\mathrm{IF}_{2}$. The transmission line $\mathrm{T}_{10}$ and capacitor $\mathrm{C}_{4}$ form a high-pass filter to pass the signal $\mathrm{LO}_{2}$ and block the low-frequency signal $\mathrm{RF}_{2} . \mathrm{T}_{11}$ is a $50-\mathrm{Ohm}$ quarterwave short-circuited transmission line at $\mathrm{IF}_{2}$ and so point $\mathrm{D}$ is seen by the signal $\mathrm{IF}_{2}$ as an open circuit and by the lowfrequency signal $\mathrm{RF}_{2}$ as a short circuit. This mixer circuit does not need any DC supply, so it is simple, low cost and easy to implement.

\section{QUADRATURE PREDISTORTER}

The wanted IM3 signals from Mixer \#2 are fed to a $90^{\circ}-$ coupler, as shown in Fig. 3, to produce an in-phase and quadrature-phase signals of $\left[\cos \left(2 \omega_{2}-\omega_{1}\right) t+\cos \left(2 \omega_{1}-\omega_{2}\right) t\right]$ and $j\left[\cos \left(2 \omega_{2}-\omega_{1}\right) t+\cos \left(2 \omega_{1}-\omega_{2}\right) t\right]$, respectively. The amplitudes and phases of the IM3 signal and the original twotone signal $\cos \left(\omega_{1} t\right)+\cos \left(\omega_{2} t\right)$ are adjusted using the corresponding attenuators and phase-shifters. The two signals are then combined to produce:

$$
\begin{aligned}
& \cos \left(\omega_{1} t+\phi_{0}\right)+\cos \left(\omega_{2} t+\phi_{0}\right) \\
& +k_{1}\left[\cos \left(\left(2 \omega_{2}-\omega_{1}\right) t+\phi_{1}\right)+\cos \left(\left(2 \omega_{1}-\omega_{2}\right) t+\phi_{1}\right)\right] \\
& +j k_{2}\left[\cos \left(\left(2 \omega_{2}-\omega_{1}\right) t+\phi_{2}\right)+\cos \left(\left(2 \omega_{1}-\omega_{2}\right) t+\phi_{2}\right)\right]
\end{aligned}
$$

where $k_{1}, k_{2}$ and $\phi_{0}, \phi_{1}, \phi_{2}$ are the adjusted amplitudes and phases, respectively, of the corresponding signal components. The resultant signal of (3) is fed to the HPA. Assume that we adjust the phase shifters such that $\phi_{0}=\phi_{1}=\phi_{2}$, then substituting (3) into (2) gives the 3rd-order IMD at the HPA output:

$$
\begin{aligned}
\operatorname{IMD}(t) & =\left(C_{1}+j C_{2}\right) \cos \left(\left(2 \omega_{1}-\omega_{2}\right) t+\phi_{0}\right) \\
& +\left(C_{3}+j C_{4}\right) \cos \left(\left(2 \omega_{2}-\omega_{1}\right) t+\phi_{0}\right)
\end{aligned}
$$

where $C_{1} \ldots C_{4}$ are functions of $k_{1}$ and $k_{2}$. Thus the powers of the IMD products can be minimized by adjusting $k_{1}$ and $k_{2}$.

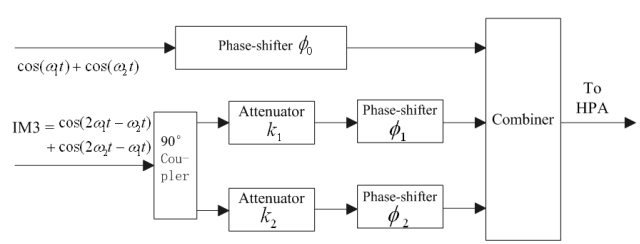

Fig. 3 Attenuators and phase shifters of predistorter

\section{RESULTS AND DISCUSSIONS}




\subsection{IM3 generator}

The IM product generator has been fabricated on a $\mathrm{PCB}$ using Roger's RO4005C. The 3-dB 90 hybrid coupler used was Anaren's model JP503S and the Schottky diodes were Avago-tech's HSMS 282X. The chip capacitors $\mathrm{C}_{1}, \mathrm{C}_{2}, \mathrm{C}_{3}$ and $\mathrm{C}_{4}$ had values of $100 \mathrm{pF}, 100 \mathrm{pF}, 10 \mathrm{nF}, 100 \mathrm{pF}$, respectively, with a mounting size of 0603 (60 mil x 30 mil). The inductor $\mathrm{L}_{1}$ had a value of $100 \mathrm{nH}$ with a mounting size of 0805 (80 mil x 50 mil).

Since the IM3 generator is designed for HPA predistortion, the unwanted spurious frequencies generated will degrade the performance of the predistorter and the conversion loss will determine whether the IM3 signals generated are large enough for use. A single-tone test and a two-tone test have been used to study the performances, in terms of conversion loss and unwanted spurious frequencies, of the IM3 generator using simulation and measurements.

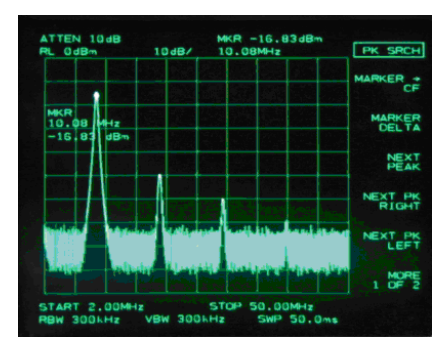

Fig. 4 Measured signal spectrum at IF\#1 of Mixer \#1 in single-tone test

\begin{tabular}{|c|c|c|}
\hline Frequency $(\mathrm{MHz})$ & $\begin{array}{c}\text { Simulated power } \\
(\mathrm{dBm})\end{array}$ & $\begin{array}{c}\text { Measured power } \\
(\mathrm{dBm})\end{array}$ \\
\hline 10 & -15.618 & -16.83 \\
\hline 20 & -54.090 & -52.04 \\
\hline 30 & -60.323 & -61.07 \\
\hline 40 & -62.237 & -70.13 \\
\hline 50 & -66.838 & Noise Floor \\
\hline
\end{tabular}

Table 1 Tone signal powers at IF\#1 of Mixer \#1 in single-tone test

In the single-tone test for Mixer \#1, the signal powers of $\mathrm{RF}_{1}$ and $\mathrm{LO}_{1}$ were set at $-10 \mathrm{dBm}$ and $5 \mathrm{dBm}$, respectively, and the corresponding frequencies were $\omega_{1}=2.21 \mathrm{GHz}$ and $\omega_{2}=$ $2.2 \mathrm{GHz}$, resulting in a difference-frequency signal $\mathrm{IF}_{1}$ at a frequency of $\left(\omega_{1}-\omega_{2}\right)=10 \mathrm{MHz}$. The measured signal spectrum at IF\#1 is shown in Fig. 4 which indicates that $\mathrm{IF}_{1}$ has a power of $-16.83 \mathrm{dBm}$, leading to a conversion loss of $6.83 \mathrm{~dB}$. Taking into account the cable loss of about $1 \mathrm{~dB}$, the actual conversion loss is in fact about $5.83 \mathrm{~dB}$. The optimum conversion loss of passive mixers is $3.92 \mathrm{~dB}$ [11], so our Mixer \#1 has a slightly additional conversion loss of about $2 \mathrm{~dB}$ more than that of an optimum mixer. Simulation using Agilent ADS 2006A has also been carried out on the mixer. For comparison purpose, the power levels of the output tones at different frequencies in both simulation and measurement are shown in Table 1. It can be seen that at the frequencies of 10,20 and $30 \mathrm{MHz}$, the differences between the simulation and measurement results are less than $2 \mathrm{~dB}$. The measurement results show that the unwanted spurious frequencies are more than $35 \mathrm{~dB}$ below that of the wanted tone at $10 \mathrm{MHz}$.

In the single-tone test for Mixer \#2, the signal $\mathrm{IF}_{1}$ with a power of $-16.83 \mathrm{dBm}$ at $10 \mathrm{MHz}$ from Mixer \#1 was fed to $\mathrm{RF} \# 2$ of Mixer \#2. The tone signal $\mathrm{LO}_{2}$ at $\mathrm{LO} \# 2$ had a frequency of $2.2 \mathrm{GHz}$ and power of $5 \mathrm{dBm}$. The expected difference signal $\mathrm{IF}_{2}$ was $2.2 \pm 0.01 \mathrm{GHz}$. The measured spectrum at the output IF\#2 of Mixer \#2 is shown in Fig. 5. It can be seen that the output wanted signals at $2.21 \mathrm{GHz}$ and $2.19 \mathrm{GHz}$ have the power levels of $-25.1 \mathrm{dBm}$ and $-26 \mathrm{dBm}$, respectively. Taking into account the $1 \mathrm{~dB}$ cable loss, the corresponding conversion losses of Mixer \#2 were $8.27 \mathrm{~dB}$ and $9.17 \mathrm{~dB}$. Table 2 shows the simulated and measured powers of different tones in IF\#2 of Mixer \#2. It can be seen that the differences between the measured and the simulation results of the wanted tones are all within $1.5 \mathrm{~dB}$. The measured results show that the strongest spurious frequencies are at $2.18 \mathrm{GHz}$ and $2.22 \mathrm{GHz}$ and both are about $25-30 \mathrm{~dB}$ lower than the wanted frequencies. The remaining spurious frequencies are close to the noise floor as shown in Fig. 5.

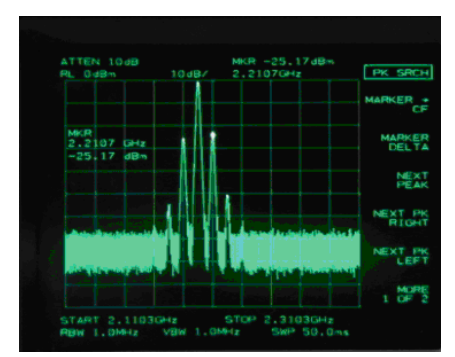

Fig. 5 Signal spectrum from IF\#2 of Mixer \#2 in single-tone test

\begin{tabular}{|c|c|c|}
\hline Frequency $(\mathrm{GHz})$ & Simulated $(\mathrm{dBm})$ & Measured $(\mathrm{dBm})$ \\
\hline 2.2 & -3.105 & -3.57 \\
\hline 2.21 & -25.432 & -25.17 \\
\hline 2.19 & -25.271 & -26.00 \\
\hline 2.22 & -50.848 & -51.50 \\
\hline 2.18 & -48.950 & -58.37 \\
\hline 2.23 & -73.984 & -65.18 \\
\hline 2.17 & -65.622 & -68.42 (noise floor) \\
\hline
\end{tabular}

Table 2 Tone signal powers at IF\#2 of Mixer \#2 in single-tone test

A two-tone test was used to study the performance of the generator on generating the IM products. In the test, a twotone signal at the frequencies of $2.21 \mathrm{GHz}\left(\omega_{1}\right)$ and $2.2 \mathrm{GHz}$ $\left(\omega_{2}\right)$ with equal amplitude was used as the input signals to

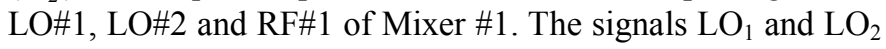
at $\mathrm{LO} \# 1$ and $\mathrm{LO} \# 2$ had a power of $5 \mathrm{dBm}$, while the signal $\mathrm{RF}_{1}$ at RF\#1 had a power of $-10 \mathrm{dBm}$. The wanted IM3 signals at the frequencies of $2 \omega_{2}-\omega_{1}$ and $2 \omega_{1}-\omega_{2}$ from IF\#2 should be at 2.19 and $2.22 \mathrm{GHz}$. The measured signal spectrum is shown in Fig. 6. Table 3 shows the measured and simulated IMD products at different frequencies. It can be seen that the IM3 signals at $2.19 \mathrm{GHz}$ and $2.22 \mathrm{GHz}$ have the powers of $24.1 \mathrm{dBm}$ and $-24.33 \mathrm{dBm}$, respectively. Again, taking into account the 1-dB cable loss, the actual output power are $23.1 \mathrm{dBm}$ and $-23.33 \mathrm{dBm}$. Since the input power of $R F_{1}$ was - 
$10 \mathrm{dBm}$, the conversion losses are $13.1 \mathrm{~dB}$ and $13.33 \mathrm{~dB}$ at 2.19 $\mathrm{GHz}$ and $2.22 \mathrm{GHz}$, respectively.

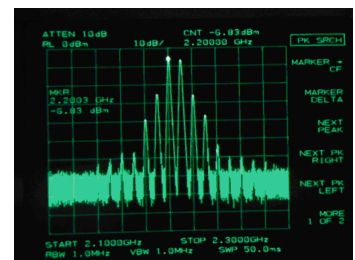

Fig. 6 Signal spectrum from IM products generator in two-tone test

It can be seen in Fig. 6 that the generator also generates other higher-order IMD products, such as the $5^{\text {th }}$ - and $7^{\text {th }}$-order IMD products, i.e., IM5 and IM7. Table 3 shows that IM3 at $3 \omega_{2}-2 \omega_{1}=2.18 \mathrm{GHz}$ and $3 \omega_{1}-2 \omega_{2}=2.23 \mathrm{GHz}$ have the powers of about $15 \mathrm{~dB}$ less than that of IM3. While IM7 at $4 \omega_{2}-3 \omega_{1}=2.17 \mathrm{GHz}$ and $4 \omega_{1}-3 \omega_{2}=2.24 \mathrm{GHz}$, have the powers of about $30 \mathrm{~dB}$ lower than that of IM3. The remaining spurious are merely several $\mathrm{dB}$ higher than the noise floor.

\begin{tabular}{|c|c|c|}
\hline Frequency $(\mathrm{GHz})$ & Simulated $(\mathrm{dBm})$ & Measured $(\mathrm{dBm})$ \\
\hline 2.20 & -5.089 & -6.83 \\
\hline 2.21 & -5.147 & -6.83 \\
\hline 2.19 & -26.173 & -24.10 \\
\hline 2.22 & -26.026 & -24.33 \\
\hline 2.18 & -39.126 & -39.67 \\
\hline 2.23 & -39.208 & -37.50 \\
\hline 2.17 & -50.208 & -57.03 \\
\hline 2.24 & -55.157 & -52.67 \\
\hline
\end{tabular}

Table 3 Tone signal powers from IM products generator in twotone test

\subsection{Predistorter}

Simulations using Agilent ADS 2006A and measurement tests have been used to assess the performance of the IM product generator for predistortion of a practical base station HPA. The characteristic of a practical base station HPA, HW1900-40 manufactured by Bravotech Inc, China, was modeled by the complex polynomial in (2) and used in ADS 2006A. The simulated and measured results of the predistorter using the IM products generator are shown in Fig. 7 and Fig. 8. In the measurement, the output powers from the HPA in these two tests were kept the same at $30 \mathrm{~W}(14.8 \mathrm{dBW})$ and the spectra with and without the predistorter were obtained via a 40-dB coupler. It can be seen from Fig. 7 that the quadrature predistorter can suppress the $3^{\text {rd }}$-order IMD output power by more than $20 \mathrm{~dB}$, compared with no predistortion. The measured result in Fig. 8 has also shown a similar improvement of $15 \mathrm{~dB}$ suppression in the $3^{\text {rd }}$-order IMD products.
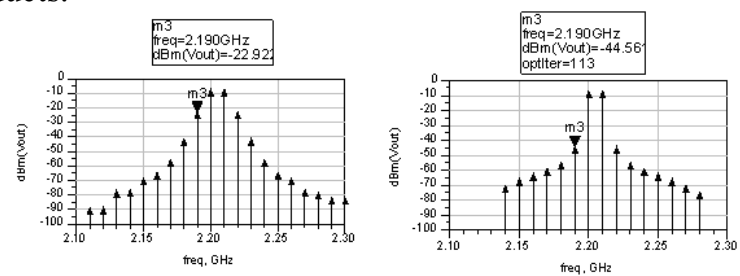

(a) without predistortion (b) with predistortion

Fig. 7 Simulated two-tone signal spectra from HPA

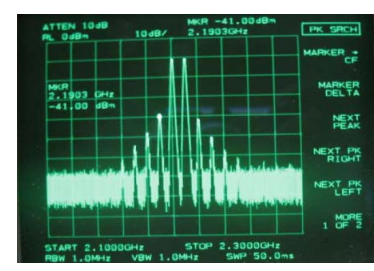

(a) without predistortion

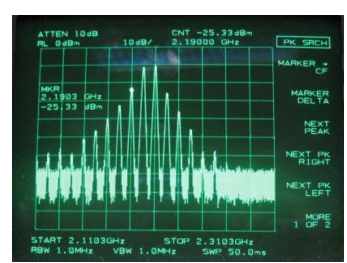

(b) with predistortion
Fig. 8 Measured two-tone signal spectra

\section{CONCLUSIONS}

This paper has presented the design of an IM products generator using simple mixers constructed with Schottky diodes for predistortion of HPAs. Simulation and measurement results have shown that the IM products generator has a low conversion loss and low spurious components, coupling with the advantages of simple structure, no DC bias and no additional filters requirement. Simulation and experimental tests on a practical HPA operating at 2.2 $\mathrm{GHz}$ with an output power of $30 \mathrm{~W}(14.8 \mathrm{dBW})$ have shown that, for a two-tone test, the predistorter can reduce the $3^{\text {rd }}$ IMD products by $15 \mathrm{~dB}$.

\section{REFERENCES}

[1] P. B. Kenington, High-linearity RF Amplifier Design, Norwood: Artech House, pp. 7-10, 2000.

[2] S.C. Cripps, RF Power Amplifiers for Wireless Communications, Norwood, MA: Artech House, pp. 254-281, 1999.

[3] S. M. Li, D. Jing, and W. S. Chan, "Verification of practicality of using the second harmonic for reducing IMD," Electron. Lett., vol. 34, no. 11, pp. 1097-1098, 1998.

[4] K. J. Cho, et al, "Multi-order predistortion of power amplifiers using a second harmonic based technique," IEEE Microwave and Wireless Components Letters, Vol. 13, pp. 452-454, Oct. 2003.

[5] M. Modeste, D. Budimir, M. Moazzam, and C. S. Aitchison, "Analysis and practical performance of a difference frequency technique for improving the multicarrier IMD performance of RF amplifiers," IEEE MTT-S Symp. Dig., pp. 53-56, Feb. 1999.

[6] T. Nesimoglu, C. N. Canagarajah, and J. P. McGeehan, "A broad band polynomial predistorter for reconfigurable radio", VTC 01, pp. 1968-1972, 2001.

[7] J. G. McRory, and R. H. Johnston, "An RF amplifier for low Inter modulation distortion," Microwave Symposium Digest, IEEE MTT-S International, pp. 1741-1744, 1994.

[8] K. Sangwon, and L. Jenshan, "A linearized cascode CMOS power amplifier," Wireless and Microwave Technology Conference, IEEE Annual, pp. 1-4, 2006.

[9] D. H. Jang, et al, "Analog predistorter using a cartesian vector modulator structure," Microwave and Optical Technology Letters, Vol. 43, No.4, pp. 343-348, Nov 20. 2004.

[10] Y. H. Liew, and J. Joe, "RF and IF ports matching circuit synthesis for a simultaneous conjugate-matching mixer using quasilinear analysis," IEEE Transactions on Microwave Theory and Technique, Vol. 50, No.9, pp. 2056-2062, 2002.

[11] G. Rowan and L. Besser, Practical RF Circuit Design for Modern Wireless Systems: Active Circuits and Systems, Vol. Boston London: Artech House, pp. 442-451, 2002. 